\title{
Uterine Corpus Carcinoma and Carcinosarcoma pT3 TNM Finding v8
}

National Cancer Institute

\section{Source}

National Cancer Institute. Uterine Corpus Carcinoma and Carcinosarcoma pT3 TNM

Finding v8. NCI Thesaurus. Code C139772.

Uterine corpus carcinoma or carcinosarcoma with tumor involving serosa, adnexa, vagina, or parametrium. (from AJCC 8th Ed.) 